Cite this: Chem. Commun., 2014, 50,5980

Received 29th March 2014 Accepted 14th April 2014

DOI: $10.1039 / c 4 c c 02346 c$

www.rsc.org/chemcomm

\section{Ethylene bis-imidazoles are highly potent and selective activators for isozymes VA and VII of carbonic anhydrase, with a potential nootropic effect $\dagger$}

\author{
Bogdan Draghici, ${ }^{a}$ Daniela Vullo, ${ }^{b}$ Suleyman Akocak, ${ }^{a}$ Ellen A. Walker, ${ }^{a}$ \\ Claudiu T. Supuran ${ }^{\star b}$ and Marc A. Ilies ${ }^{\star a}$
}

\begin{abstract}
A series of ethylene bis-imidazoles was synthesized via a novel microwave-mediated synthesis. Biological testing on eight isozymes of carbonic anhydrase (CA) present in the human brain revealed compounds with nanomolar potency against CA VA and CA VII, also displaying excellent selectivity against other CA isozymes present in this organ.
\end{abstract}

The dynamic state of all organisms relates directly to their metabolic activity. Carbon dioxide is the end product of catabolic processes of higher vertebrates and its rapid transport and elimination are critical for achieving fast metabolic rates. Carbonic anhydrases (E.C. 4.2.1.1) are zinc enzymes that catalyze the reversible hydration of $\mathrm{CO}_{2}$ under physiological conditions: $\mathrm{CO}_{2}+\mathrm{H}_{2} \mathrm{O} \leftrightarrow \mathrm{HCO}_{3}{ }^{-}+\mathrm{H}^{+}$. Through the activity of mitochondrial isozymes, especially CA VA, cytosolic isozymes CA I, CA II, CA VII and membrane-bound isozymes CA IV, CA IX, CA XII, CA XIV, carbon dioxide can be rapidly hydrated and transported from mitochondria through the cytoplasm towards the exterior of the cell (Fig. 1). ${ }^{1}$ About $85 \%$ of total $\mathrm{CO}_{2}$ produced in the body is transported as $\mathrm{HCO}_{3}{ }^{-}$to lungs. The above-mentioned CA isozymes are particularly important for the brain ${ }^{2}$ which is a major $\mathrm{CO}_{2}$ producer and where the $\mathrm{CO}_{2}$ / $\mathrm{HCO}_{3}{ }^{-}$buffer constitutes the main buffer in the interstitial/extracellular space. ${ }^{3}$ These CA isozymes actively neutralize the steep $\mathrm{pH}$ gradients (as high as $0.1 \mathrm{pH}$ units) generated by neuronal discharge that are superimposed on the normal flux of protons caused by aerobic metabolism. ${ }^{3}$ Strict $\mathrm{pH}$ control maintains membrane polarization and the proper ionization (hence the action) of various key neurotransmitters, such as glutamate, GABA, and dopamine. Fluctuation of local $\mathrm{pH}$ has been shown to affect receptor responses

\footnotetext{
${ }^{a}$ Department of Pharmaceutical Sciences and Moulder Center for Drug Discovery Research, Temple University School of Pharmacy, 3307 N Broad Street, Philadelphia, PA-19140, USA. E-mail: mailies@temple.edu; Fax: +1 215-707-5620; Tel: +1 215-707-1749

${ }^{b}$ NEUROFARBA Department, Pharmaceutical Sciences Section, Universita degli Studi di Firenze, Polo Scientifico, Via Ugo Schiff no. 6, 50019 Sesto Fiorentino, Florence, Italy. E-mail: claudiu.supuran@unifi.it; Fax: +39-055-4573385; Tel: $+39-055-4573005$

$\dagger$ Electronic supplementary information (ESI) available. See DOI: 10.1039/c4cc02346c
}

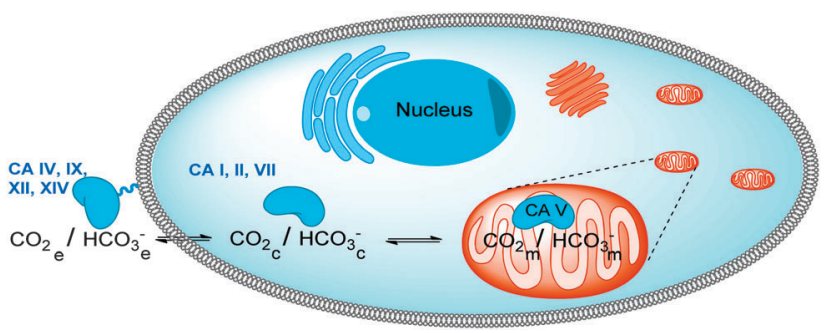

Fig. 1 Schematics of $\mathrm{CO}_{2}$ flux, from mitochondria to the cytoplasm and to the exterior of the cell, revealing that the CA isozymes involved in the process and the three different $\mathrm{CO}_{2} / \mathrm{HCO}_{3}{ }^{-}$pools (extracellular (e), cytosolic (c) and mitochondrial (m)) equilibrated by them.

such as the NMDA receptor. ${ }^{4}$ CAs are directly involved in cerebrospinal fluid (CSF) secretion at the level of the choroid plexus, generating bicarbonate from $\mathrm{CO}_{2}$ and $\mathrm{H}_{2} \mathrm{O}$, and regulating through ion hydration the volume, buffering properties, and turnover of CSF. ${ }^{5}$

The key role played by CAs in brain homeostasis and normal cerebral function is emphasized by CA deficiency syndrome - a distinct genetic disorder characterized by severe pathological modifications of the brain, lungs, kidneys, and bone metabolism. ${ }^{6}$ Patients with this disorder lack CA II in these tissues/organs and blood and display a range of symptoms including cerebral calcification, respiratory and renal tubular acidosis, inherited osteopetrosis, and mental retardation. ${ }^{6}$ Moreover, in the neurodegenerative disorder of memory and cognitive function, Alzheimer's disease, the level of CA is significantly diminished. ${ }^{7}$ A similar decrease in the expression level of CA was observed in the brain of older rats relative to young animals, ${ }^{8}$ being associated with a reduced resistance to dehydration, alteration in choroidal control of brain homeostasis, and reduced cerebrospinal fluid production.

Therefore, activation of the CAs present in the brain represents a promising and rather unexplored approach to increase brain activity, especially memory and learning, with significant potential benefits in older individuals or in patients affected by Alzheimer's disease and other forms of dementia. Sun and Alkon first suggested that carbonic anhydrase activators (CAAs) can enhance learning 
and memory, thus eliciting a nootropic effect. ${ }^{9}$ For example, in the hidden-platform water maze, rats injected with the CAAs phenylalanine or imidazole have significantly higher latencies to escape. In further support of the role of CA in this learning model, the CA inhibitor acetazolamide impaired memory and prevented the improvements in learning observed after injection of both phenylalanine and imidazole. ${ }^{9}$

Memory improvements in rodents have also been observed through CA activation with the dipeptide carnosine. ${ }^{10}$ Finding potent and selective CAAs with a pharmacokinetic profile that could ensure good brain penetrability represents the critical next step towards the generation of a potential nootropic effect.

CA activators act by speeding up the deprotonation of zinc bound water (the rate-determining step, eqn (2) in the catalytic mechanism), with the generation of the active form of the enzyme: ${ }^{11}$

$$
\begin{aligned}
\mathrm{EZn}^{2+}-\mathrm{OH}^{-}+\mathrm{CO}_{2} & \rightleftharpoons \mathrm{EZn}^{2+}-\mathrm{HCO}_{3}^{-} \\
& \stackrel{+\mathrm{H}_{2} \mathrm{O}}{\rightleftharpoons} \mathrm{EZn}^{2+}-\mathrm{OH}_{2}+\mathrm{HCO}_{3}{ }^{-}
\end{aligned}
$$

$\mathrm{EZn}^{2+}-\mathrm{OH}_{2} \rightleftharpoons \mathrm{EZn}^{2+}-\mathrm{OH}^{-}+\mathrm{H}^{+}$-rate determining step

In the presence of an activator $\mathrm{A}$, eqn (2) becomes (3): ${ }^{11}$

$$
\begin{aligned}
& \mathrm{EZn}^{2+}-\mathrm{OH}_{2}+\mathrm{A} \rightleftharpoons\left[\mathrm{EZn}^{2+}-\mathrm{OH}_{2}-\mathrm{A}\right] \underset{\text { enzyme-activator complexes }}{\rightleftharpoons} \\
& {\left[\mathrm{EZn}^{2+}-\mathrm{OH}^{-}-\mathrm{AH}^{+}\right] } \rightleftharpoons\left[\mathrm{EZn}^{2+}-\mathrm{OH}^{-}-\mathrm{AH}^{+}\right] \\
& \rightleftharpoons \mathrm{EZn}^{2+}-\mathrm{OH}^{-}+\mathrm{AH}^{+}
\end{aligned}
$$

Comprehensive structure-activity relationship (SAR) studies done by us or by others ${ }^{11}$ revealed that efficient CA activators possess a proton-shuttling group attached to a hydrophobic/amphiphilic aromatic/heterocyclic anchor via a short, flexible linker (Chart 1, inset). We identified imidazole as a very potent proton shuttling moiety, in combination with various anchors (Chart 1). In the study of CA activation with histamine 1, X-ray crystallography revealed that the activator is anchored at the entrance of the active site cavity through the amino group. The imidazole moiety participates in shuttling protons between the active site and the bulk solvent, thus acting as a second proton shuttle of the enzyme. This study demonstrated the superiority of the imidazole structural unit $\left(\mathrm{p} K_{\mathrm{a}}=6-7\right)$ against the primary amino moiety $\left(\mathrm{p} K_{\mathrm{a}}=9-10\right)$ as a proton-shuttling group. ${ }^{12}$ Activation of CA with bis-azoles such as bis-imidazole 2 was discovered almost simultaneously. In these $\mathrm{N}$-alkylated bis-azoles 2 , one imidazole ring acts as a hydrophobic anchor and the other one as a proton shuttle (Chart 1). ${ }^{13}$ Subsequent studies performed by Silverman's group on activation of CA V using histidine analogues 4-bromoethylimidazole (4-BEI, 3), 2-chloromethylimidazole (2-CMI), 4-chloromethyl-imidazole (4-CMI, 4) as covalent CA activators confirmed the efficiency of imidazoles as CA activators. ${ }^{14} \mathrm{An}$ extensive SAR study on pyridinium azoles by our team ${ }^{15}$ confirmed the imidazole moiety as the best proton shuttle (e.g., compound 5), due to its $\mathrm{p} K_{\mathrm{a}}$ in the maximum activity range for most CA isozymes (6.5-8.5 $\mathrm{p} K_{\mathrm{a}}$ units). The study demonstrated the efficiency of the pyridinium moiety to anchor the activator at the rim of the active site. ${ }^{15}$ Subsequent CA activation studies with various imidazole derivatives (4-methylimidazole $6, \mathrm{~L}$ - and D-histidine $(7,8)$, carnosine 9 and congeners), and with the 4-(pyridinium-ethyl)imidazoles 10-12 recently synthesized by our groups confirmed this conclusion. ${ }^{16-18}$ The CAA design approach was supported by site-directed mutagenesis studies and X-ray crystal structures of the complexes of various CA isozymes with imidazole-based activators. ${ }^{17-19}$

The positively-charged molecules 5 and 10-12 were shown to generate membrane-impermeant CA activators useful for studying the physiology of membrane-bound CA isozymes. Moreover, the same studies ${ }^{15,18}$ revealed that small differences in the structure of activators 10-12 could trigger dramatic changes in the activation profile of these compounds through the exploitation of the differences in the amino acids lining the active site of various isozymes. Very recently, McKenna's team revealed the existence of several binding pockets for imidazole CAAs such as 4-methylimidazole 6 and its isomers. ${ }^{21}$ Encouraged by the very promising results obtained with CAAs of type 10-12, we decided to investigate compact bisimidazoles 13 in which two imidazole moieties were C-linked via an ethyl linker, attached to the 4(5)-position of the imidazole ring. In these new CAAs, one imidazole moiety acts as the proton-shuttling moiety, while the other imidazole anchors the activator at the rim of the active site, being structurally related to their congeners 2 but having the full proton shuttling capabilities conferred by a free imidazole NH. Hydrophobic substituents of increased steric bulk were placed in the 2-position of the imidazole ring in order

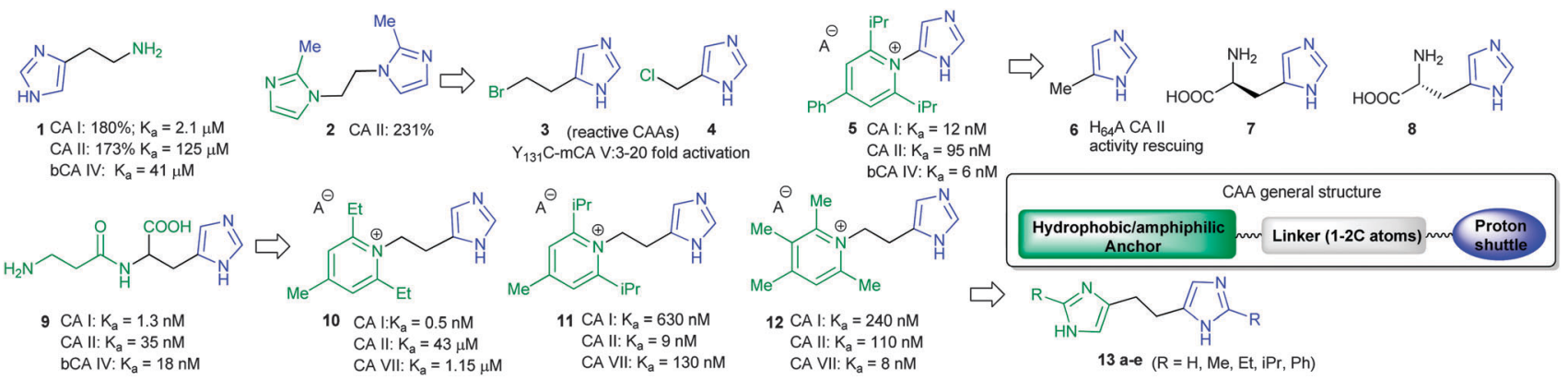

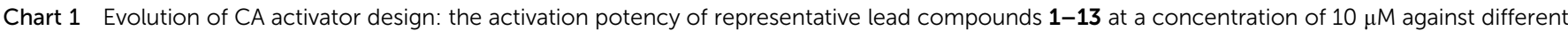

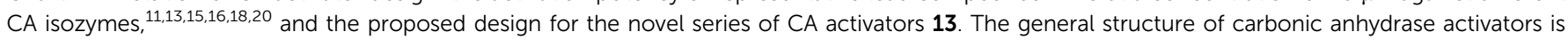
shown in the inset. 
to facilitate the anchoring of the activator on the rim of the different isozymes of CA. These substituents also increase lipophilicity of the bis-imidazoles 13, thus facilitating the crossing of the blood-brain barrier which is critical for achieving the nootropic effect.

In terms of synthesis, bis[1 $H$-imidazol-4(5)yl]alkanes can be prepared from $\alpha$-dihaloalkyldione and formamide at elevated temperatures $\left(180{ }^{\circ} \mathrm{C}\right)$ via Bredereck cyclization. ${ }^{22}$ However, this method is limited to systems in which the two $\alpha$-keto units in the molecule are separated by four to eight $\mathrm{C}$ atoms, with longer spacers generating higher yields. A better option for the synthesis of bis-imidazoles of type 13 having short alkyl spacers is the strategy of Schunack et al., ${ }^{23}$ who prepared them through the condensation of amidines with $\alpha$-dihaloalkyldione in the presence of ammonia. Since this method requires an autoclave and generates only moderate yields even after $20 \mathrm{~h}$ reaction time, we tested a microwave-mediated procedure based on this synthetic strategy.

The novel MW procedure allows the achievement of high temperatures and moderate pressures, decreasing the reaction time significantly. Using this procedure, we were able to generate bis[1 $H$-imidazol-4(5)yl]ethane derivatives 13a-e, substituted at position 2 of the imidazole ring, from 1,6-dibromohexane-2,5dione $15^{24}$ and corresponding alkylamidines $16^{25}$ in the presence of ammonia solution in methanol (Scheme 1, ESI $\dagger$ ).

Bis-imidazoles 13a-e were subsequently tested for the activation of eight CA isozymes involved in brain homeostasis, namely the mitochondrial hCA VA, the cytosolic hCA I, hCA II, hCA VII and the membrane-bound hCA IV, hCA IX, hCA XII and hCA XIV, using the $\mathrm{CO}_{2}$ hydration assay method (Table 1 , see $\mathrm{ESI} \dagger$ for details).

Data from Table 1 revealed an excellent activation profile for the new compounds. For all isozymes we identified activators that were more potent than histamine 1, the prototype CA

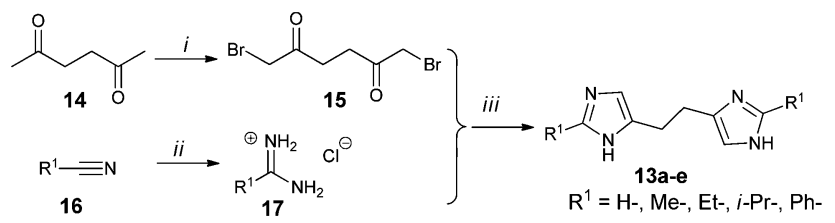

Scheme 1 Synthesis of bis[1 $\mathrm{H}$-imidazol-4(5)yl]ethane derivatives 13a-e Reactions and conditions: (i) $\mathrm{Br}_{2}, \mathrm{H}_{2} \mathrm{SO}_{4}, \mathrm{MeOH}, 0{ }^{\circ} \mathrm{C}$ to r.t., $12 \mathrm{~h}$; (ii) $\mathrm{HCl}$, EtOH $0{ }^{\circ} \mathrm{C}$ to r.t., $2 \mathrm{~h}$ then $\mathrm{NH}_{3} 7 \mathrm{~N}, \mathrm{MeOH} 4 \mathrm{~h}$ r.t.; (iii) $\mathrm{NH}_{3}-\mathrm{MeOH} 7 \mathrm{~N}, \mathrm{MW}$ $200 \mathrm{~W}, 120^{\circ} \mathrm{C}, 40 \mathrm{~min}$.

Table 1 Activation profile of human CA isozymes (hCAs) with bisimidazoles 13a-e

\begin{tabular}{lcllllllll}
\hline & \multicolumn{1}{l}{$K_{\mathrm{A}}(\mu \mathrm{M})$} & & & & & & \\
\cline { 2 - 9 } & hCA & hCA & hCA & hCA & \multicolumn{1}{l}{ hCA } & \multicolumn{2}{ll}{ hCA } & hCA & hCA \\
No. & I & II & IV & VA & VII & IX & XII & XIV \\
\hline Histamine & 2.1 & 125 & & & 37.5 & & & \\
13a & 16.4 & 68.5 & 1.25 & 0.021 & 0.015 & 9.51 & 8.63 & 13.9 \\
13b & 23.5 & 69.3 & 4.51 & 0.009 & 0.020 & 13.1 & 5.40 & 10.2 \\
13c & 1.13 & 76.8 & 2.37 & 0.037 & 0.071 & 24.7 & 6.13 & 18.5 \\
13d & 4.81 & 80.4 & 9.50 & 0.131 & 0.054 & 20.1 & 9.85 & 24.6 \\
13e & 5.96 & 78.7 & 12.4 & 0.052 & 0.089 & 18.5 & 12.6 & 25.9
\end{tabular}

activator within this class of compounds, thus validating our new design.

The most susceptible isozymes activated with bis-imidazoles $\mathbf{1 3}$ were the mitochondrial isozyme CA VA and the brain cytosolic isozyme CA VII, followed by membrane-bound hCA IV, hCA XII, hCA IX and hCA XIV. The cytosolic isozymes hCA I and hCA II were the least susceptible to be activated with this class of compounds.

Importantly, in the case of hCA VA and hCA VII, we were able to identify activators with nanomolar potency. For these isozymes the increase in steric bulk of the substituents placed in position 2 of the imidazole rings generally caused a decrease in the potency of the activators, probably due to the limited space available in the active site and the decreased mobility of the heterocyclic rings substituted with bulky substituents such as $i \operatorname{Pr}($ 13d) and $\mathrm{Ph}$ (13e). The most potent activators were 13a $(\mathrm{R}=\mathrm{H})$, with a $K_{\mathrm{A}}=21 \mathrm{nM}$ for hCA VA, $K_{\mathrm{A}}=15 \mathrm{nM}$ for hCA VII and 13b $\left(\mathrm{R}=\mathrm{CH}_{3}\right)$ with a $K_{\mathrm{A}}=$ $9 \mathrm{nM}$ for hCA VA and $K_{\mathrm{A}}=20 \mathrm{nM}$ for hCA VII. Importantly, these activators were three orders of magnitude more selective for hCA VA and hCA VII as compared with the other cytosolic and membranebound CA isozymes tested (Table 1).

Thus, bis-imidazoles 13 displayed only micromolar potency against membrane-bound hCA IV, hCA XII, hCA IX and hCA XIV and cytosolic isozymes hCA I and hCA II. Moreover, for these isozymes the increase in steric bulk of substituents placed in position 2 of the imidazole rings in compounds 13 was not dramatically decreasing the activation potency. This particular activation profile may suggest a different binding location of bis-imidazoles $\mathbf{1 3}$ in the active sites of hCA VA and hCA VII as compared with the other CA isozymes tested.

The recent identification of multiple binding sites for differently substituted imidazoles within the active site of hCA $\mathrm{II}^{21}$ supports this hypothesis. Work is in progress to shed light on the structural origin of the potency and selectivity of these novel CA activators.

In conclusion, we are proposing a novel set of nanomolar CA activators that act selectively on CA VA and CA VII, key CA isozymes involved in brain metabolism. Their potency and selectivity against other CA isozymes present at the level of the brain, together with their optimum lipophilicity recommend them as novel probes for elucidation of the role of CA VA and CA VII in brain physiology and potential modulators of learning, memory and cognition.

This work was supported by TUSP Dean's Office and an EU Grant (Dynano).

\section{Notes and references}

1 C. T. Supuran, Nat. Rev. Drug Discovery, 2008, 7, 168; V. Alterio, A. Di Fiore, K. D'Ambrosio, C. T. Supuran and G. De Simone, Chem. Rev., 2012, 112, 4421.

2 S. Parkkila, EXS, 2000, 79; C. K. Tong, L. P. Brion, C. Suarez and M. Chesler, J. Neurosci., 2000, 20, 8247; G. N. Shah, B. Ulmasov, A. Waheed, T. Becker, S. Makani, N. Svichar, M. Chesler and W. S. Sly, Proc. Natl. Acad. Sci. U. S. A., 2005, 102, 16771.

3 M. Chesler, Physiol. Rev., 2003, 83, 1183.

4 S. Makani and M. Chesler, J. Neurosci., 2007, 27, 7438.

5 T. H. Maren, C. W. Conroy, G. C. Wynns and D. R. Godman, J. Pharmacol. Exp. Ther., 1997, 280, 98.

6 W. S. Sly and P. Y. Hu, Annu. Rev. Biochem., 1995, 64, 375; W. S. Sly, D. Hewett-Emmett, M. P. Whyte, Y. S. Yu and R. E. Tashian, Proc. Natl. Acad. Sci. U. S. A., 1983, 80, 2752. 
7 W. Meier-Ruge, P. Iwangoff and K. Reichlmeier, Arch. Gerontol. Geriatr., 1984, 3, 161.

8 C. Masseguin, S. LePanse, B. Corman, J. M. Verbavatz and J. Gabrion, Neurobiol. Aging, 2005, 26, 917.

9 M. K. Sun and D. L. Alkon, J. Pharmacol. Exp. Ther., 2001, 297, 961; M. K. Sun and D. L. Alkon, Trends Pharmacol. Sci., 2002, 23, 83.

10 A. R. Hipkiss, J. Alzheimer's Dis., 2007, 11, 229.

11 C. T. Supuran and A. Scozzafava, EXS, 2000, 197; M. Ilies, A. Scozzafava and C. T. Supuran, in Carbonic Anhydrase - its Inhibitors and Activators, ed. C. T. Supuran, A. Scozzafava and J. Conway, CRC Press, Boca Raton, 2004, p. 317; C. Temperini, A. Scozzafava and C. T. Supuran, in Drug Design of Zinc-Enzyme Inhibitors - Functional, Structural, and Disease Applications, ed. C. T. Supuran and J. Y. Winum, Wiley, Hoboken, 2009, p. 473.

12 F. Briganti, S. Mangani, P. Orioli, A. Scozzafava, G. Vernaglione and C. T. Supuran, Biochemistry, 1997, 36, 10384.

13 C. T. Supuran, A. T. Balaban, P. Cabildo, R. M. Claramunt, J. L. Lavandera and J. Elguero, Biol. Pharm. Bull., 1993, 16, 1236; C. T. Supuran, R. M. Claramunt, J. L. Lavandera and J. Elguero, Biol. Pharm. Bull., 1996, 19, 1417.

14 J. N. Earnhardt, S. K. Wright, M. Qian, C. Tu, P. J. Laipis, R. E. Viola and D. N. Silverman, Arch. Biochem. Biophys., 1999, 361, 264.

15 M. Ilies, M. D. Banciu, M. A. Ilies, A. Scozzafava, M. T. Caproiu and C. T. Supuran, J. Med. Chem., 2002, 45, 504.
16 A. Scozzafava and C. T. Supuran, J. Med. Chem., 2002, 45, 284.

17 D. Duda, C. Tu, M. Qian, P. Laipis, M. Agbandje-McKenna, D. N. Silverman and R. McKenna, Biochemistry, 2001, 40, 1741; I. Elder, C. Tu, L. J. Ming, R. McKenna and D. N. Silverman, Arch. Biochem. Biophys., 2005, 437, 106; C. Temperini, A. Scozzafava, D. Vullo and C. T. Supuran, Chem. - Eur. J., 2006, 12, 7057.

18 K. Dave, A. Scozzafava, D. Vullo, C. T. Supuran and M. A. Ilies, Org. Biomol. Chem., 2011, 9, 2790; K. Dave, M. A. Ilies, A. Scozzafava, C. Temperini, D. Vullo and C. T. Supuran, Bioorg. Med. Chem. Lett., 2011, 21, 2764.

19 K. M. Jude, S. K. Wright, C. Tu, D. N. Silverman, R. E. Viola and D. W. Christianson, Biochemistry, 2002, 41, 2485.

20 C. T. Supuran, A. Dinculescu and A. T. Balaban, Rev. Roum. Chim., 1993, 38, 343; C. T. Supuran, M. Barboiu, C. Luca, E. Pop, M. E. Brewster and A. Dinculescu, Eur. J. Med. Chem., 1996, 31, 597; M. A. Ilies, M. D. Banciu, M. Ilies, F. Chiraleu, F. Briganti, A. Scozzafava and C. T. Supuran, Eur. J. Med. Chem., 1997, 32, 911.

21 M. Aggarwal, B. Kondeti, C. Tu, M. Maupin, D. N. Silverman and R. McKenna, IUCrJ, 2014, 1, 129.

22 B. Miller, J. Altman and W. Beck, Synthesis, 1997, 347.

23 C. Leschke, J. Altman and W. Schunack, Synthesis, 1993, 197.

24 C. Meziere, M. Fourmigue, E. Canadell, R. Clerac, K. Bechgaard and P. Auban-Senzier, Chem. Mater., 2000, 12, 2250.

25 P. L. Barker, P. L. Gendler and H. Rapoport, J. Org. Chem., 1981, 46, 2455. 